\title{
Chloroquine Used in Combination with Chemotherapy Synergistically Suppresses Growth and Angiogenesis In Vitro and In Vivo
}

\author{
EBRU GUREL-GUREVIN ${ }^{1}$, HULYA TUBA KIYAN ${ }^{2}$, OSMAN BEHZAT BURAK ESENER ${ }^{3}$, SEYMA AYDINLIK ${ }^{4}$, \\ AYCA UVEZ ${ }^{3}$, ENGIN ULUKAYA ${ }^{5}$, KONSTANTINOS DIMAS ${ }^{6}$ and ELIF ILKAY ARMUTAK ${ }^{3 *}$ \\ ${ }^{1}$ Department of Biology, Faculty of Science and ${ }^{3}$ Department of Histology and Embryology, \\ Faculty of Veterinary Medicine, Istanbul University, Istanbul, Turkey; \\ ${ }^{2}$ Department of Pharmacognosy, Faculty of Pharmacy, Anadolu University, Eskisehir, Turkey; \\ ${ }^{4}$ Department of Molecular Biology, Faculty of Science, Uludag University, Bursa, Turkey; \\ ${ }^{5}$ Department of Medical Biochemistry, Faculty of Medicine, Istinye University, Istanbul, Turkey; \\ ${ }^{6}$ Department of Pharmacology, School of Health Science, Thessaly University, Larissa, Greece
}

\begin{abstract}
Background: The inhibition of autophagy using pharmacological inhibitors such as chloroquine may be an effective strategy to overcome chemotherapy or resistance to anti-angiogenic therapy. Materials and Methods: The cytotoxic effect of doxorubicin (0.1-1 $\mu \mathrm{M})$, chloroquine (0.25-32 $\mu \mathrm{M})$ and their combination were investigated by employing ATP assay in human umbilical vein endothelial cells (HUVECs). The effect of doxorubicin and chloroquine combination was also measured using tube formation assay on Matrigel. The anti-angiogenic activities of doxorubicin $(2.5 \mu \mathrm{g} /$ pellet $)$ and chloroquine (15 $\mu \mathrm{g} /$ pellet), their combination, and standards (50 $\mu \mathrm{g} /$ pellet) were tested in vivo using the chick embryo chorioallantoic membrane (CAM) assay. Results: The combination of doxorubicin and chloroquine significantly had a stronger anti-angiogenic effect than the positive control

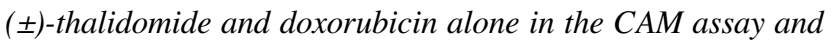
in vitro tube-formation assay. Conclusion: Chloroquine enhanced the anti-angiogenic effect of doxorubicin on CAM at the tested concentrations.
\end{abstract}

Doxorubicin, a first-generation anthracycline, is currently one of the most active cytotoxic, chemotherapeutic agents for treating various kinds of cancer such as breast and prostate cancer, childhood solid tumors, leukemia, soft-tissue

Correspondence to: Elif Ilkay Armutak, Ph.D., Department of Histology and Embryology, Faculty of Veterinary Medicine, University of Istanbul, 34320, Avcilar, Istanbul, Turkey. E-mail: elif@istanbul.edu.tr, elifarmutak@gmail.com

Key Words: Doxorubicin, chloroquine, angiogenesis, CAM assay, tube formation. sarcoma, aggressive lymphomas and human hepatocellular carcinoma (1-4). It has anticancer activity by altering topoisomerase II activity, as well as inhibiting DNAdependent DNA and RNA synthesis through multiple mechanisms including intercalation with DNA strands and generation of reactive oxygen species (ROS) $(4,5)$. This topoisomerase II-mediated DNA damage is followed by growth arrest in $G_{1}$ and $G_{2}$ and programmed cell death (3).

Despite its highly potent anticancer activity against various tumor cell types, doxorubicin has a low therapeutic index and, due to its associated acute and chronic toxicities including myelosupression, immunosupression, and cardioand renal toxicity, its clinical use in cancer treatment is limited. Therefore, lowering its toxicity by combining this agent with another highly effective novel non-toxic agent, it is still a high priority need. Besides reducing the side-effects of doxorubicin, a combination treatment might also modulate different signaling pathways in cancer cells $(4,6)$. In this context, various drugs and molecules, such as the lipidlowering agent probucol (7), all-trans retinoic acid (4), noscapine (6), zoledronic acid (8), coenzyme Q10-liquid crystalline nanoparticles (5), SU54 16 (9) and anti-angiogenic agents such as sirolimus (10), tetrathiomolybdate (11), combretastatin A4 (12), in combination with doxorubicin were reported to lead to enhanced antitumor efficacy.

Chloroquine, a well-known anti-malarial drug, is used in the clinic to treat auto-immune diseases such as lupus erythematosus and rheumatoid arthritis by inhibiting the immune system and due to its anti-inflammatory properties; it is also used for retroviral purposes $(13,14)$. It is known to show potent cytotoxic effects against various cancer cell types such as human cervical, colorectal, lung, breast, and hepatocellular and mouse colon cancer cells $(15,16)$. 
The anticancer properties of chloroquine are due to its ability to induce cell-cycle arrest, inhibit autophagy and promote apoptosis in tumor cells $(14,16,17)$. As a lysosomotropic agent, chloroquine senzitizes various cancer cells to the antiproliferative effect of radiation, chemotherapy, cisplatin, 5-fluorouracil and oxaliplatin by inhibiting lysosomal acidification $(16,18)$. Autophagy, a conserved catabolic process that includes degradation of cytoplasmic proteins and organelles for further intracellular recycling, plays a controversial role in tumorigenesis due to its protective or degenerative properties in tumor cells during tumor progression (19). Dysregulation of autophagy may occur in a manner that enables tumor cell survival even in a limited-nutrient environment depending on cancer type (18). Chloroquine has already been reported to restore drug sensitivity and increase the intracellular targets of drugs by inhibiting the degradation of autophagic cargo and by blocking autophagic flux (17). Because of its highly safe profile as compared to other autophagy inhibitors, chloroquine might be more appropriate for use in combinatorial cancer therapy with other chemotherapeutics as a cancer-specific chemosensitizer $(15,16)$.

Angiogenesis, the formation of new blood vessels from existing ones, plays a vital role in embryo growth, wound healing, post-ischemic tissue restoration and endometrial changes during the menstrual cycle physiologically, but also acts in inflammation, several autoimmune diseases such as lupus erythematosus, and cancer $(13,20)$. In this light, we aimed to investigate doxorubicin and chloroquine for their anti-angiogenic properties alone and in combination using the chicken chorioallantoic membrane (CAM) assay. The in vivo CAM assay is very useful, well-established and an animal alternative method for screening angiogenic, antiangiogenic and irritation potency of substances as well as therapeutics $(21,22)$. To the best of our knowledge this is the first study on the angiogenic evaluation of doxorubicin and chloroquine combination using the CAM assay.

\section{Materials and Methods}

Cell culture and chemicals. Human umbilical vein endothelial cells (HUVECs) were purchased from the American Type Culture Collection (Manassas, VA, USA) and cultured in EBM-2 endothelial cell basal medium (Lonza, Walkersville, MD, USA) with full supplements endothelial growth medium-2 (EGM-2) bullet kit : $2 \%$ fetal bovine serum (FBS), $0.4 \%$ human fibroblast growth factor-2 (hFGF-2), $0.1 \%$ vascular endothelial growth factor (VEGF), $0.1 \%$ recombinant analog of insulin-like growth factor-1 (R3-IGF-1), $0.1 \%$ human epidermal growth factor (hEGF), $0.04 \%$ hydrocortisone, $0.1 \%$ ascorbic acid, $0.1 \%$ heparin, $0.1 \%$ gentamicin, amphotericin b) $(\mathrm{GA}-1000)$ at $37^{\circ} \mathrm{C}$ in a humidified atmosphere containing $5 \% \mathrm{CO}_{2}$. Chloroquine (Sigma, St. Louis, MO, USA) was dissolved in deionized water at a concentration of $50 \mathrm{mM}$ as a stock solution; reference anti-angiogenics (prednisone, cortisone, and racemic $( \pm)$-thalidomide; Sigma-Aldrich Chemie Gmbh Munich,
Germany) were dissolved in dimethylsulfoxide (DMSO) as a stock solution $(500 \mathrm{mM})$ and doxorubicin was dissolved in Molecular Biology water at $2 \mathrm{mg} / \mathrm{ml}$ concentration as a stock solution. The highest concentration of DMSO did not exceed $0.1 \%(\mathrm{v} / \mathrm{v})$ in the cell culture. Both doxorubicin (Kocak Farma, Turkey) and chloroquine aliquots were stored at $-20^{\circ} \mathrm{C}$ for futher use and any further dilutions were made in culture medium.

ATP viability assay. The cytotoxic effects of doxorubicin, chloroquine and their combination on HUVECs were examined with an ATP assay. Briefly, cells were seeded at a density of $5 \times 10^{3}$ cells per well of 96-well plates in triplicate and incubated overnight. After 24-h incubation, cells were pretreated with chloroquine $(0.25-32 \mu \mathrm{M})$ for $24 \mathrm{~h}$ prior to a 48 -h treatment with doxorubicin $(0.1-1 \mu \mathrm{M})$. Cells were also treated with reference anti-angiogenics (cortisone, thalidomide, prednisone) at concentrations of 0.1-400 $\mu \mathrm{M}$ for $48 \mathrm{~h}$. Reference anti-angiogenics were prepared in DMSO (final concentration of dimethyl sulfoxide, $0.1 \%$ ).

In order to carry out the ATP assay, at the end of the 48-h treatment period, $150 \mu \mathrm{l}$ of medium was removed from each well and $50 \mu \mathrm{l}$ of somatic cell ATP-releasing agent (ATP Bioluminescent Somatic Cell Assay Kit; Sigma, Steinheim, Germany) was added. After mixing thoroughly, the microplate was allowed to stand for $30 \mathrm{~min}$ at room temperature. At the end of the incubation, $50 \mu \mathrm{l}$ of mixture from each well was transferred to a white non-translucent plate. To each well of the plate, $50 \mu$ of ATP Assay Mix (ATP Bioluminescent Somatic Cell Assay Kit, Sigma, Steinheim, Germany) was added and the emitted light was measured using a 96-well Microplate Luminometer (Bio-Tek, Winooski, VT, USA). ATP viability assays were performed at least twice and the results are given as mean $\pm \mathrm{SD}$ of independent experiments.

Tube formation assay. The effect of doxorubicin and chloroquine combination on morphogenesis of endothelial cells was evaluated using the tube formation assay on Matrigel (BD Biosciences, Road Billerica, MA, USA). Briefly, $50 \mu$ l Matrigel was added to each well of pre-chilled 96 -well plate and incubated at $37^{\circ} \mathrm{C}$ for $30 \mathrm{~min}$ to polymerize the Matrigel. Cells at a density of $2 \times 10^{4}$ cells/well were seeded on a Matrigel-pre-coated 96-well plate and treated with doxorubicin, chloroquine alone or their combination at different concentrations for $2,6,12,16 \mathrm{~h}$. DMSO and reference antiangiogenics were included as negative and positive controls, respectively. Tube formation was checked quantitatively under an inverted microscope.

CAM assay. Fertilized eggs were obtained from Has Tavuk Company (Bursa and Sivrihisar, Turkey). Agarose was purchased from Invitrogen (Thermo Fisher Scientific Waltham, MA, USA) and sodium dodecyl sulphate (SDS) from Fluka-Biochemika (FlukaBiochemika, St Louis, MO, USA).

Doxorubicin $(0.25 \mathrm{mg} / \mathrm{ml})$, chloroquine $(1.5 \mathrm{mg} / \mathrm{ml})$ and standards $(5 \mathrm{mg} / \mathrm{ml})$ were dissolved in a $2.5 \%(\mathrm{w} / \mathrm{v})$ agarose solution. For ease of application, pellets of these solutions $(10 \mu \mathrm{l})$ were prepared by applying dropwise onto circular stainless-steel supports of $5 \mathrm{~mm}$ diameter, cooling to room temperature for solidification and subsequently applying onto CAMs.

The in vivo CAM assay was performed as described previously by our research group (23). Fertilized hen eggs previously incubated for $72 \mathrm{~h}$ at $36.5^{\circ} \mathrm{C}$ and at a relative humidity of $80 \%$ were positioned in a horizontal position and rotated several times. Albumin (8-10 ml) 
Gurel-Gurevin et al: Anti-Angiogenic Effects of Chloroquine and Doxorubicin Combination

Table I. Semi-quantitative scoring system of anti-angiogenic effects on chick embryo chorioallantoic membrane (CAM) after treatment (23, 24).

\begin{tabular}{|c|c|c|}
\hline Scale & Anti-angiogenic effect & Effects observed on CAM after treatment \\
\hline$<0.5$ & Inactive & No change (normal embryo growth). \\
\hline $0.5-0.75$ & Weak & No capillary-free area. \\
\hline$>0.75-1$ & Strong & $\begin{array}{l}\text { Area with reduced density of capillaries around the pellet not larger than its own area. } \\
\text { Small capillary-free area or area with significantly reduced density of capillaries. } \\
\text { Effects not larger than double the size of the pellet. }\end{array}$ \\
\hline$>1$ & Very strong & Capillary-free area around the pellet at least double the size of the pellet. \\
\hline
\end{tabular}

Formula used for scoring: Average score $=[$ Number of eggs (score $>1) \times 2+$ number of eggs (score $>0.75-1) \times 1] /($ total number of eggs scored)

was aspirated from a small hole punched with a injector needle in the bottom of the egg. Then a hole on the top of the egg shell of 2 $\mathrm{cm}$ diameter was opened by forceps. The cavity in the egg shell was covered with stretch film and eggs were incubated at $36.5^{\circ} \mathrm{C}$ at a relative humidity of $80 \%$ for another $72 \mathrm{~h}$. Pellets ( 1 pellet/egg) were placed on the CAM which had approximately a diameter of $2 \mathrm{~cm}$ and the egg shell hole again covered with stretch film. Twenty-four hours later, the eggs were evaluated under a stereo-microscope (Leica, Wetzlar, Germany) with Leica Application Suite, Las V4.7 (Leica); 10-15 eggs were utilized for each test condition (controls, doxorubicin or chloroquine alone or their combination). For all conditions, three independent experiments were performed.

Anti-angiogenic effects were evaluated by using a scoring system (see Table I) $(23,24)$. The anti-angiogenic scores were calculated using the following formula: Average score $=[$ Number of eggs (score $>1$ ) $\times 2+$ number of eggs (score $>0.75-1) \times 1] /$ (total number of eggs scored).

As positive controls prednisone, cortisone, thalidomide and negative control SDS at $50 \mu \mathrm{g} / \mathrm{pellet}$ were also tested. CAMs treated with solidified agarose-solution in pellet form $(2.5 \%, \mathrm{w} / \mathrm{v})$ were also tested as a blank. Tests for each compound were performed in triplicate.

Statistical analysis. Statistically analysis was performed using oneway ANOVA followed by Tukey's multiple comparison test with Graphpad Prism 6.0 (GraphPad Software, La Jolla, CA, USA) statistical software for Windows. $p$-Values of 0.05 or less were considered statistically significant. Results are expressed as mean \pm standard deviation.

\section{Results}

Anti-growth effect of doxorubicin and chloroquine combination by ATP assay. The anti-growth effect of doxorubicin and chloroquine combination was investigated against HUVECs by ATP assay. The viability upon chloroquine treatment was determined after treatment with a concentration range of 0.25 to $32 \mu \mathrm{M}$ for $24 \mathrm{~h}$ (Figure 1A). In the same manner, the viability of cells upon doxorubicin treatment was assessed after treatment with different concentrations $(0.1-1 \mu \mathrm{M})$ of doxorubicin for $48 \mathrm{~h}$ (Figure 1B). Results show that the doxorubicin exhibited an antigrowth effect in a dose-dependent manner. On the basis of these growth curves, the stronger growth-inhibitory doses of doxorubicin $(0.4,0.5,0.8,1 \mu \mathrm{M})$ were selected and combined with different doses of chloroquine (Figure 2). The results showed significant decrease in cell viability especially at $0.8 \mu \mathrm{M}$ doxorubicin plus $4 \mu \mathrm{M}$ chloroquine and $1 \mu \mathrm{M}$ doxorubicin plus $8 \mu \mathrm{M}$ chloroquine treatment groups ( $p<0.05$ and $p<0.01$, respectively).

Cells were also treated with reference anti-angiogenics (cortisone, thalidomide, and prednisone) at concentrations of 0.1-400 $\mu \mathrm{M}$ for $48 \mathrm{~h}$. Reference anti-angiogenics did not demonstrate any cytotoxic effect on HUVECs after 48-h treatment (Figure 3), except cortisone and prednisone at 400 $\mu \mathrm{M}$ dose. As shown in Figure 4, control HUVECs, plated on Matrigel and incubated with control medium, were able to form capillary-like structures while reference antiangiogenics used as positive control slightly reduced tube formation in a dose-dependent manner (Figure 4A). Doxorubicin blocked tube formation both at 0.8 and $1 \mu \mathrm{M}$ doses within $16 \mathrm{~h}$; however, chloroquine had no effect on tube formation at 4 or $8 \mu \mathrm{M}$ after $16 \mathrm{~h}$ (Figure 4B). In contrast, treatment with chloroquine at $4 \mu \mathrm{M}$ combined with $1 \mu \mathrm{M}$ doxorubicin, and at $8 \mu \mathrm{M}$ with 0.8 or $1 \mu \mathrm{M}$ doxorubicin significantly reduced tube formation after $16 \mathrm{~h}$ (Figure 4C).

Anti-angiogenic effects of doxorubicin, chloroquine and their combination. The anti-angiogenic activities of doxorubicin, chloroquine and their combination were examined using the CAM assay. Prednisone, cortisone, and thalidomide were used as positive controls, and SDS was employed as negative control for the assay. The results of evaluation of the antiangiogenic effect are summarized in Table II.

According to the microscopic evaluations (Figure 5), the combination of doxorubicin at $2.5 \mu \mathrm{g} /$ pellet and chloroquine at $15 \mu \mathrm{g} /$ pellet showed very strong anti-angiogenic effect with a score better than that of doxorubicin alone. On the contrary, chloroquine alone showed no anti-angiogenic effect compared to the positive controls prednisone, cortisone and thalidomide at $50 \mu \mathrm{g} /$ pellet. Importantly, doxorubicin and 
A

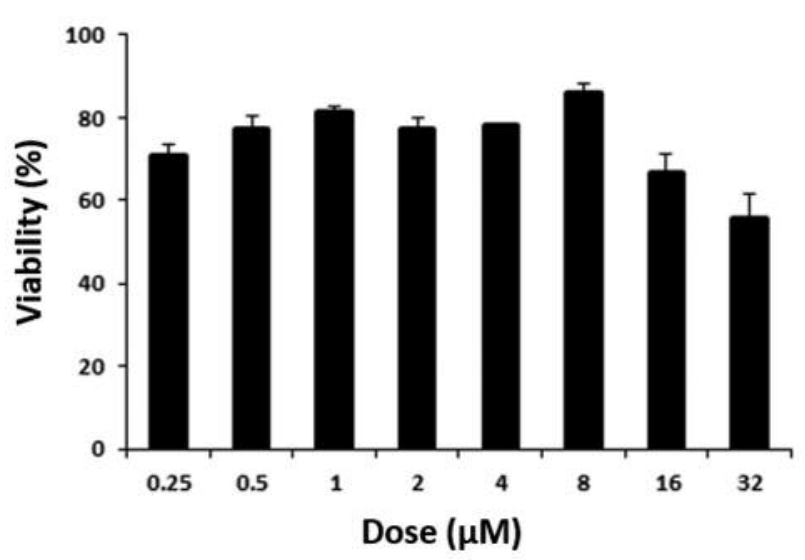

B

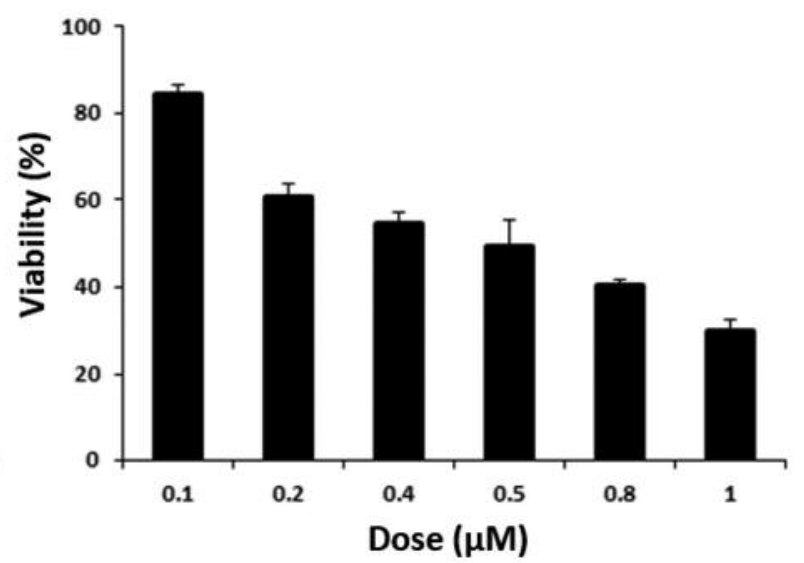

Figure 1. Assessment of viability of human umbilical vein endothelial cells after treatment with different doses of chloroquine for $24 \mathrm{~h}(\mathrm{~A})$ and doxorubicin for $48 h(B)$ using the ATP assay. The results are representative of two independent experiments.

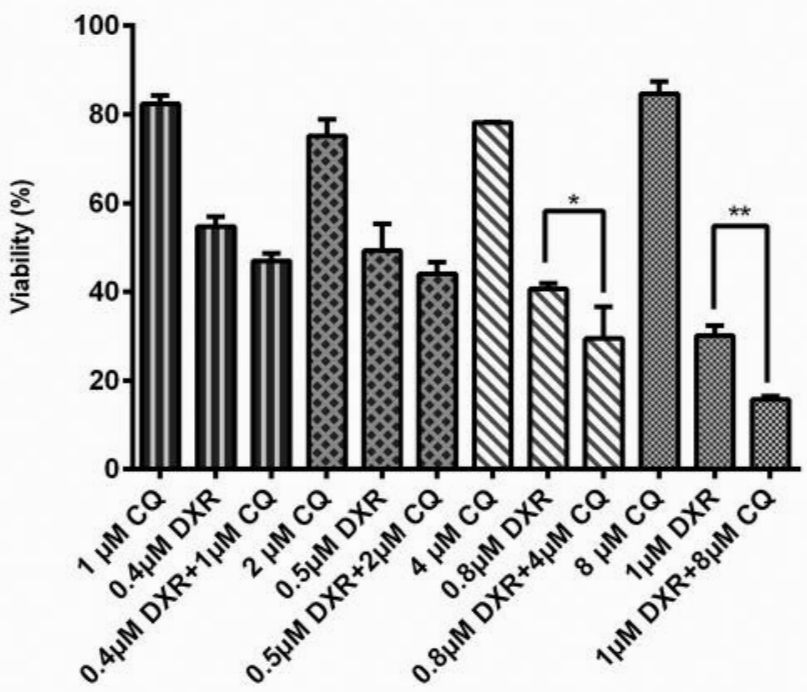

Figure 2. The anti-growth effects of combinatorial treatment with chloroquine $(C Q)$ and doxorubicin (DXR) on human umbilical vein endothelial cells were measured using the ATP assay after $48 \mathrm{~h}$ treatment. Statistically significant different at: $* p<0.05$ and $* * p<0.01$.

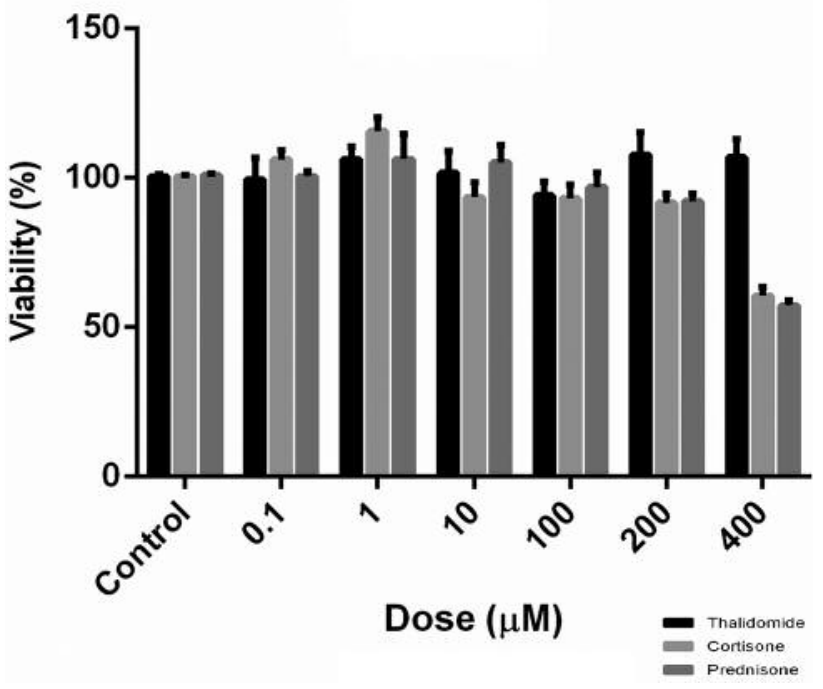

Figure 3. Determination of viability of human umbilical vein endothelial cells after $48 \mathrm{~h}$ treatment with reference anti-angiogenics by ATP assay. The data were obtained via two independent experiments. chloroquine combination did not lead to membrane irritation nor toxicity at the tested concentration.

\section{Discussion}

There has been considerable interest in the potential antitumor effects of therapies combining chemotherapeutic agents and autophagy inhibitors for the treatment of cancers (25).

In this study, we showed that doxorubicin in combination with chloroquine exhibited significant anti-growth effect against HUVECs in a dose-dependent manner. Amongst all combinations tested, the combination of doxorubicin at a concentration of $1 \mu \mathrm{M}$ and chloroquine at a concentration of 


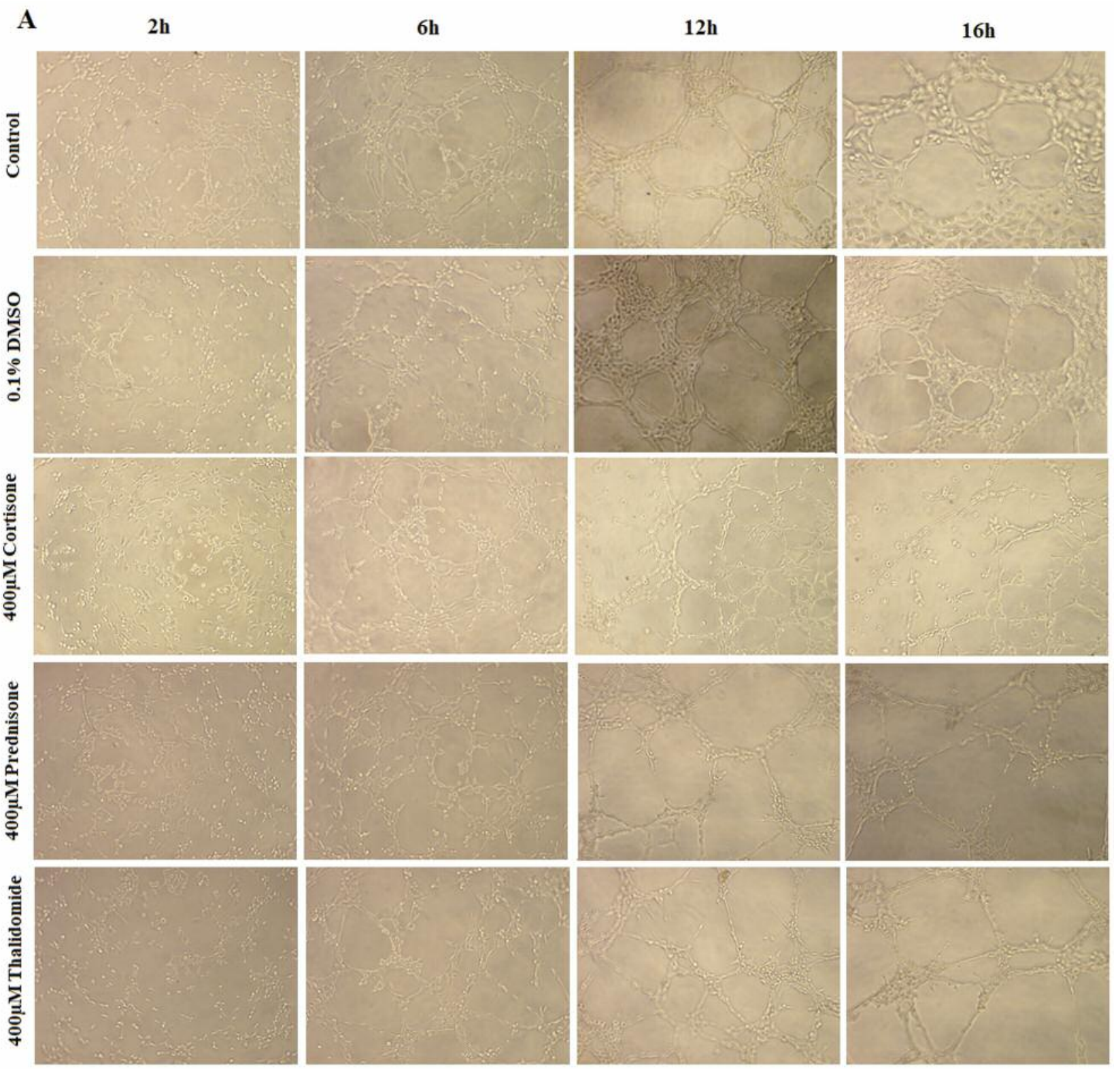

Figure 4. Continued

$8 \mu \mathrm{M}$ for $48 \mathrm{~h}$ was found to be the most effective (Figure 1). The results regarding the concentration of doxorubicin seem to be in good agreement with the clinically relevant concentrations of the drug. Effective doses of doxorubicin used in this study were similar to those used in other studies, which ranged from $0.1 \mu \mathrm{M}$ to $25 \mu \mathrm{M}$ for HUVECs $(26,27)$. More importantly the use of the autophagy inhibitory drug in combination with doxorubicin resulted in slightly better activity compared to doxorubicin alone.
It is well established that HUVECs are organized into lumen-like structures when cultured on Matrigel (23, 28-30). Therefore, we further examined the anti-angiogenic effects of doxorubicin, chloroquine and their combination on tube formation in HUVECs.

HUVECs cultures were treated with different concentrations of chloroquine, doxorubicin or their combination. A previous study demonstrated chloroquine inhibited tube networks of HUVECs (20-40 $\mu \mathrm{M})$ and also 


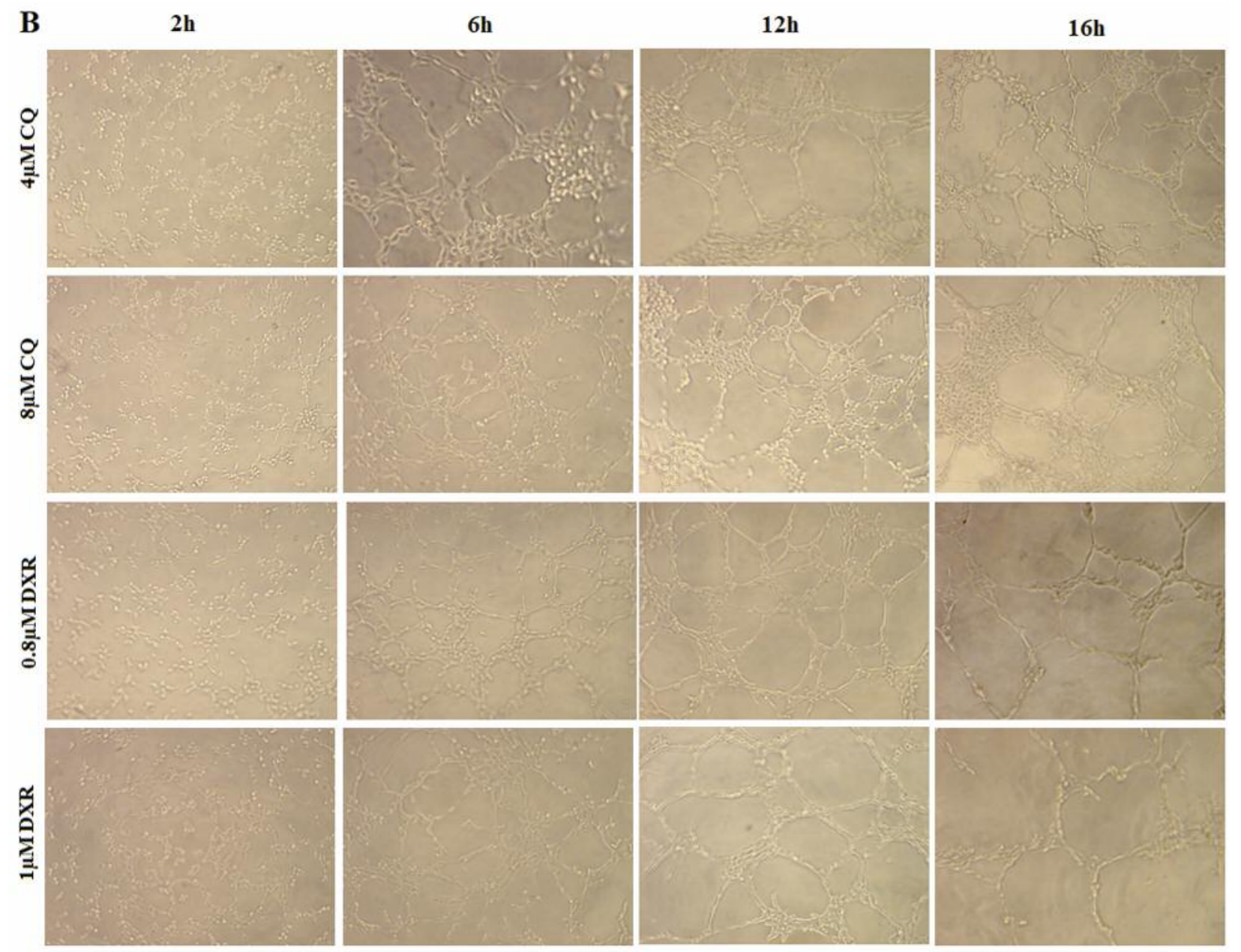

Figure 4. Continued

restrained angiogenesis in the CAM $(10 \mathrm{mg} / \mathrm{ml})$ at higher doses. In this experiment, chloroquine was used at lower doses in both tube formation $(4-8 \mu \mathrm{M})$ and CAM assay (1.5 $\mathrm{mg} / \mathrm{ml}$ ) and failed to block formation of tube-like structures even after $16 \mathrm{~h}$ of incubation (31). Unlike chloroquine, treatment with doxorubicin resulted in a dose-dependent inhibition of capillary tube formation compared to vehicle control (0.1\% DMSO). This was more prominent at $16 \mathrm{~h}$ post doxorubicin addition. Moreover, at all combination doses HUVECs formed significantly fewer and thinner tubes compared to the corresponding doses of doxorubicin and chloroquine alone (Figure 4).

Anti-angiogenic therapy aimed at blocking new blood vessel formation in pathologies such as tumour or inflammatory diseases such as rheumatoid arthritis are currently emerging. Angiogenesis associated with tumor growth is believed to promote the proliferation, invasion and metastasis of cancer cells. Multiple growth factors involved in angiogenesis, including VEGF, have also been demonstrated to play a vital role in cancer progression and metastasis (32). Inhibition of angiogenesis is currently emerging as a novel probably more potent alternative approach to developing cytotoxic agents for cancer treatment, and among these findings, researchers are working on developing new anti-angiogenic strategies $(33,34)$.

Recent preclinical and clinical evidence has indicated the important role that angiogenesis plays in tumor progression $(20,35,36)$. Therefore, inhibition of angiogenesis is thought to be an important strategy in suppressing tumor growth. In order to better resemble the in vivo conditions, the CAM model was used to investigate the anti-angiogenic effects of doxorubicin and chloroquine. Data presented herein demonstrate that the two agents can be used in combination to inhibit angiogenesis. Such a combination could clearly be 

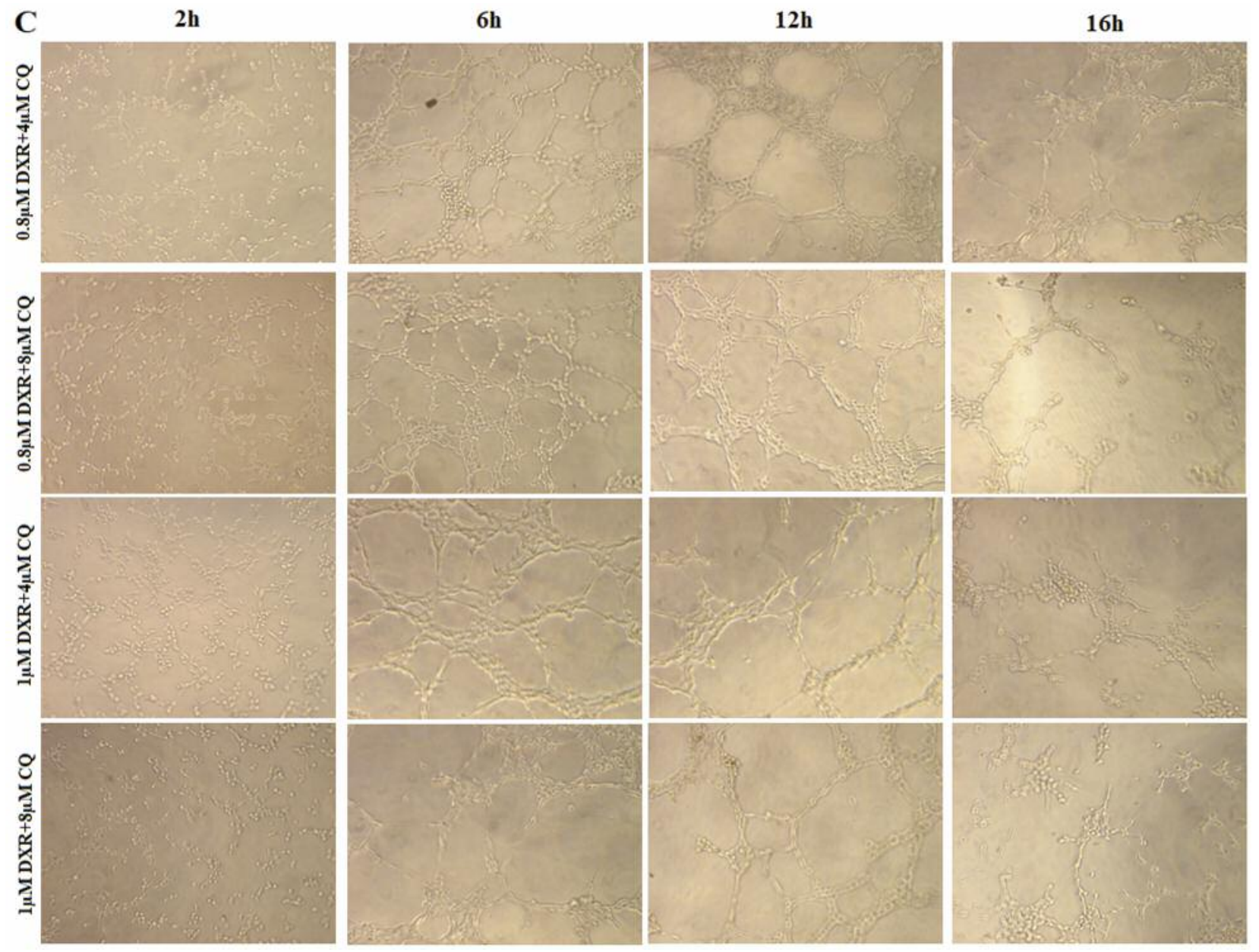

Figure 4. Micrographs showing the effect of reference anti-angiogenics $(A)$, doxorubicin $(D X R)$ and chloroquine $(C Q)$ as single agents $(B)$ and their combination $(C)$ on tube formation by human umbilical vein endothelial cells (HUVECs). The networks of tube-like structures were photographed at 2, 6, 12, 16 h following the addition of HUVECs to Matrigel. In CQ and DXR combinatorial-treated groups, tube formation was reduced compared to both agents alone in a time- and dose-dependant manner.

Table II. Anti-angiogenic effects, positive controls, doxorubicin and chloroquine alone and their combination in the chick embryo chorioallantoic membrane assay.

\begin{tabular}{|c|c|c|c|c|}
\hline Sample & $\begin{array}{l}\text { Concentration } \\
(\mu \mathrm{g} / \mathrm{pellet})\end{array}$ & Average score ${ }^{a}$ & $\begin{array}{l}\text { Anti-angiogenic } \\
\text { effect }\end{array}$ & $\begin{array}{c}\text { Irritation } \\
(\%)\end{array}$ \\
\hline Doxorubicin & 2.5 & $1.2 \pm 0.3^{*}$ & Very strong & $17 \pm 4$ \\
\hline Chloroquine & 15 & $0.4 \pm 0.1$ & Inactive & \\
\hline Doxorubicin + chloroquine & $2.5+15$ & $1.5 \pm 0.6^{*}$ & Very strong & \\
\hline Agar (blank) & $2.5 \%, \mathrm{w} / \mathrm{v}$ & $0.2 \pm 0.2$ & Inactive & - \\
\hline Prednisone (positive control) & 50 & $0.9 \pm 0.7$ & Strong & - \\
\hline Cortisone (positive control) & 50 & $1.2 \pm 0.2$ & Very strong & - \\
\hline Thalidomide (positive control) & 50 & $0.9 \pm 0.2$ & Strong & - \\
\hline Sodium dodecyl sulfate (negative control) & 50 & $0.1 \pm 0.1$ & Inactive & $87 \pm 5$ \\
\hline
\end{tabular}

aValues are mean average score \pm standard deviation ( $\mathrm{n}=45$ for the test substances and $\mathrm{n}=30$ for standards). Significantly different at $* p<0.05$ compared to positive control thalidomide. ${ }^{b}$ The irritation $(\%)$ means the percentage of the eggs in which irritation is observed. 

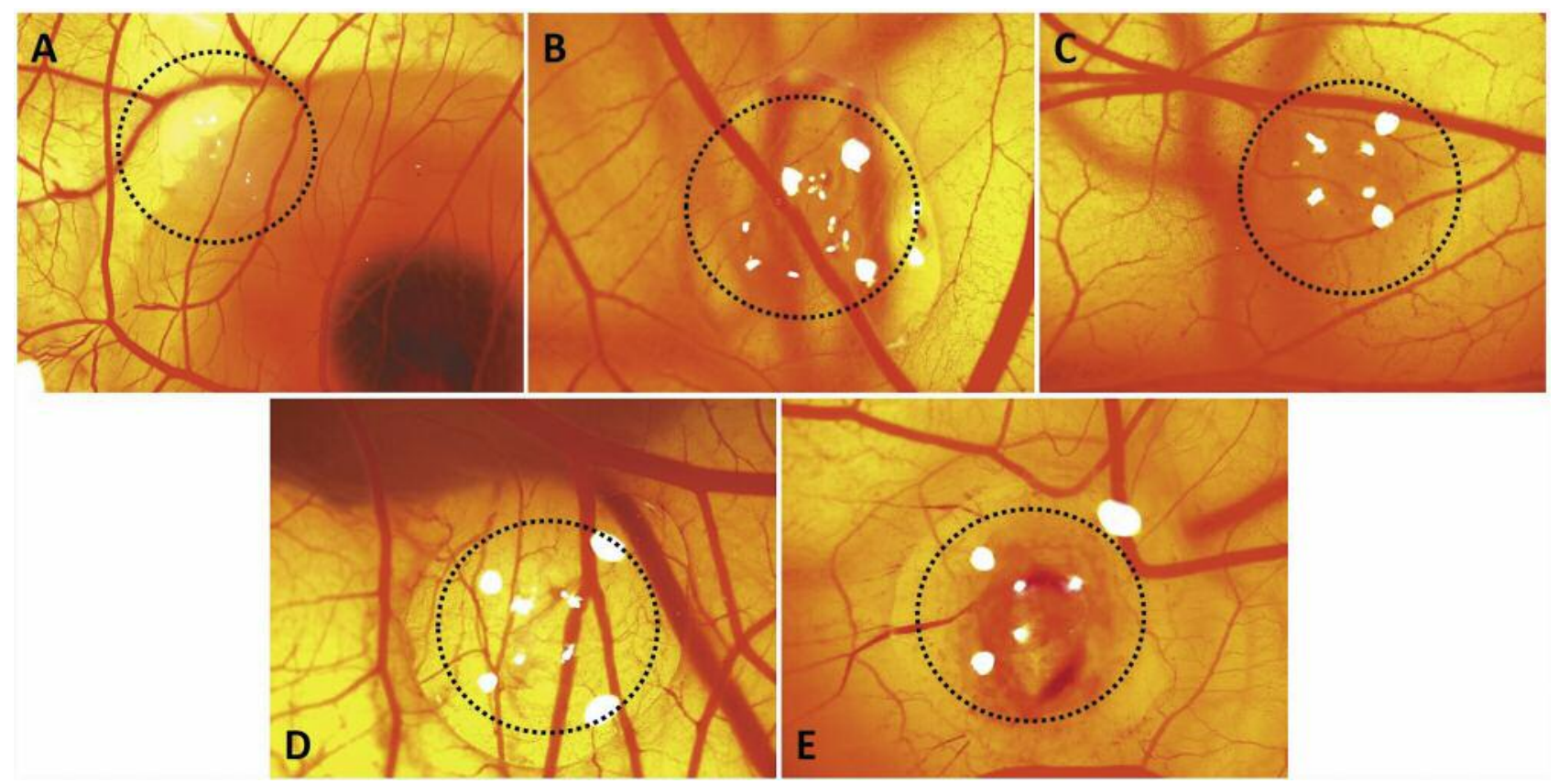

Figure 5. Assay results for chick embryo chorioallantoic membrane (CAM) treated with: A: $50 \mu \mathrm{g} /$ pellet thalidomide; B: $50 \mu \mathrm{g} / \mathrm{pellet}$ cortisone; C: $2.5 \mu \mathrm{g} /$ pellet doxorubicin; $D: 15 \mu \mathrm{g} /$ pellet chloroquine; $E: 2.5 \mu \mathrm{g} /$ pellet doxorubicin with $15 \mu \mathrm{g} /$ pellet chloroquine. While doxorubicin alone had a strong anti-angiogenic, chloroquine was ineffective. The combination of doxorubicin and chloroquine had a very strong anti-angiogenic effect.

advantageous is it may enable the use of lower doses of doxorubicin, which has important side-effects such as cardiotoxicity ( a dose limiting toxicity) while improving the efficiency of this important chemotherapeutic (37).

The antitumor anthracyclines doxorubicin, daunorubicin and epirubicin have been reported to inhibit angiogenesis by reducing vascular density and collagenous protein biosynthesis in the CAM. Tube formation by HUVECs on Matrigel has also been shown to be inhibited in the presence of these anthracyclines (38).

Chloroquine has already been reported to enhance anticancer activity in combination therapy with many therapeutic agents such as 5-fluorouracil (19) and lidamycin (39). Our results are in accordance with findings that when chloroquine combined with sunitinib, not only increased apoptotic response, but also cause reduced angiogenic response compared to treatment with each compound alone (16). Importantly, chloroquine enhances delivery to and efficacy of chemotherapeutic agent on the tumor vessel (40). Hence we assumed that chloroquine facilitates overcoming angiogenesis by combination with chemotherapeutic agent.

According to the results for anti-angiogenic activity obtained under the experimental conditions tested herein, we conclude that combination of doxorubicin with chloroquine showed synergistic activity in the CAM assay, at least partially, because of the inhibitory effect of chloroquine on of autophagy in the CAM. These results also suggest that chloroquine enhances the anti-angiogenic activity of doxorubicin.

Anti-angiogenic therapy and angioprevention, including targeting angiogenesis for the treatment or even for prevention of cancer, inflammation and of such pathologies, looks very promising for the development of new or improved anti-angiogenics (41). To the best of our knowledge, the present study is the first to evaluate the antiangiogenic of the combination of doxorubicin and chloroquine. The data obtained herein suggest that this combination warrants further thorough investigation, and of course animal experiments for proof-of-concept of a novel promising combination in cancer therapy.

\section{Conflicts of Interest}

The Authors declare that they have no confict of interest in regard to this study.

\section{Acknowledgements}

The Authors would like to thank especially Mrs. Emine Kırcalı Kocabaş from Has Tavuk Company for providing the fertilized hen eggs. This work was supported by Scientific Research Project Coordination Unit of Istanbul University. Project numbers: 49841, 55089, 21160, 20856, 49997 and 53382. 


\section{References}

1 Liu F, Tan G, Li J, Dong X, Krissansen GW and Sun X: Gene transfer of endostatin enhances the efficacy of doxorubicin to suppress human hepatocellular carcinomas in mice. Cancer Sci 98(9): 1381-1387, 2007.

2 Peled M, Shaish A, Greenberger S, Katav A, Hodish I, BenShushan, D, Barshack I, Mendel I, Frishman L, Tal R, Bangio L, Breitbart E and Harats D: Antiangiogenic systemic gene therapy combined with doxorubicin administration induced caspase 8 and 9-mediated apoptosis in endothelial cells and an anti-metastasis effect. Cancer Gene Ther 15(8): 535-542, 2008.

3 Polyak D, Ryppa C, Eldar-Boock A, Ofek P, Many A, Licha K, Kratz F and Satchi-Fainaro R: Development of PEGylated doxorubicin- $E$-[c(RGDfK $\left.)_{2}\right]$ conjugate for integrin-targeted cancer therapy. Polym Adv Technol 22(1): 103-113, 2011.

4 Zhang T, Xiong H, Dahmani FZ, Sun L, Li Y, Yao L, Zhou J and Yao J: Combination chemotherapy of doxorubicin, all-trans retinoic acid and low molecular weight heparin based on selfassembled multi-functional polymeric nanoparticles. Nanotechnology 26(14): 145101, 2015.

5 Swarnakar NK, Thanki K and Jain S: Enhanced antitumor efficacy and counterfeited cardiotoxicity of combinatorial oral therapy using doxorubicin- and coenzyme Q10-liquid crystalline nanoparticles in comparison with intravenous adriamycin Nanomedicine 10(6): 1231-1241, 2014.

6 Chougule MB, Patel AR, Jackson T and Singh M: Antitumor activity of noscapine in combination with doxorubicin in triplenegative breast cancer. PLoS One 6(3): e17733, 2011.

7 Takemura G and Fujiwara H: Doxorubicin-induced cardiomyopathy: from the cardiotoxic mechanisms to management Prog Cardiovasc Dis 49(5): 330-352, 2007.

8 Ottewell PD, Brown HK, Jones M, Rogers TL, Cross SS, Brown NJ, Coleman RE and Holen I: Combination therapy inhibits development and progression of mammary tumours in immunocompetent mice. Breast Cancer Res Treat 133(2): 523 536,2012

9 Overmoyer B, Fu P, Hoppel C, Radivoyevitch T, Shenk R, Persons M, Silverman P, Robertson K, Ziats NP, Wasman JK, Abdul-Karim FW, Jesberger JA, Duerk J, Hartman P, Hanks S, Lewin J, Dowlati A, McCrae K, Ivy P and Remick SC: Inflammatory breast cancer as a model disease to study tumor angiogenesis: results of a phase IB trial of combination SU5416 and doxorubicin. Clin Cancer Res 13(19): 5862-5868, 2007.

10 Piguet AC, Semela D, Keogh A, Wilkens L, Stroka D, Stoupis C, St-Pierre MV and Dufour JF: Inhibition of mTOR in combination with doxorubicin in an experimental model of hepatocellular carcinoma. J Hepatol 49(1): 78-87, 2008.

11 Pan Q, Bao LW, Kleer CG, Brewer GJ and Merajver SD: Antiangiogenic tetrathiomolybdate enhances the efficacy of doxorubicin against breast carcinoma. Mol Cancer Ther 2(7): 617-622, 2003.

12 Yang T, Wang Y, Li Z, Dai W, Yin J, Liang L, Ying X, Zhou S, Wang J, Zhang $X$ and Zhang Q: Targeted delivery of a combination therapy consisting of combretastatin A4 and lowdose doxorubicin against tumor neovasculature. Nanomedicine 8(1): 81-92, 2012.

13 Lesiak A, Narbutt J, Kobos J, Kordek R, Sysa-Jedrzejowska A, Norval $M$ and Wozniacka A: Systematic administration of chloroquine in discoid lupus erythematosus reduces skin lesions via inhibition of angiogenesis. Clin Exp Dermatol 34(5): 570$575,2009$.

14 Zhang Y, Liao Z, Zhang LJ and Xiao HT: The utility of chloroquine in cancer therapy. Curr Med Res Opin 31(5): 10091013, 2015.

15 Joshi P, Chakraborti S, Ramirez-Vick JE, Ansari ZA, Shanker V, Chakrabarti $P$ and Singh SP: The anticancer activity of chloroquine-gold nanoparticles against MCF-7 breast cancer cells. Colloids Surf B Biointerfaces 95: 195-200, 2012.

16 Abdel-Aziz AK, Shouman S, El-Demerdash E, Elgendy M and Abdel-Naim AB: Chloroquine synergizes sunitinib cytotoxicity via modulating autophagic, apoptotic and angiogenic machineries. Chem Biol Interact 217: 28-40, 2014.

17 Pellegrini P, Strambi A, Zipoli C, Hägg-Olofsson M, Buoncervello M, Linder S and De Milito A: Acidic extracellular $\mathrm{pH}$ neutralizes the autophagy-inhibiting activity of chloroquine: implications for cancer therapies. Autophagy 10(4): 562-571, 2014.

18 Lakhter AJ, Sahu RP, Sun Y, Kaufmann WK, Androphy EJ, Travers JB and Naidu SR: Chloroquine promotes apoptosis in melanoma cells by inhibiting BH3 domain-mediated PUMA degradation. J Invest Dermatol 133(9): 2247-2254, 2013.

19 Choi JH, Yoon JS, Won YW, Park BB and Lee YY: Chloroquine enhances the chemotherapeutic activity of 5-fluorouracil in a colon cancer cell line via cell cycle alteration. APMIS 120(7): 597-604, 2012.

20 Ribatti D: History of Research on Tumor Angiogenesis. Springer, Italy, pp 1-17, 2009.

21 Cimpean AM., Ribatti D and Raica M: The chick embryo chorioallantoic membrane as a model to study tumor metastasis. Angiogenesis 11(4): 311-319, 2008.

22 Lokman NA, Elder AS, Ricciardelli C and Oehler MK: Chick chorioallantoic membrane (CAM) assay as an in vivo model to study the effect of newly identified molecules on ovarian cancer invasion and metastasis. Int J Mol Sci 13(8): 9959-9970, 2012.

23 Ikitimur-Armutak EI, Gurel-Gurevin E, Kiyan HT, Aydinlik S, Yilmaz VT, Dimas K and Ulukaya E: Anti-angiogenic effect of a palladium(II)-saccharinate complex of terpyridine in vitro and in vivo. Microvasc Res 109: 26-33, 2017.

24 Kiyan HT, Demirci B, Başer KH and Demirci F: The in vivo evaluation of anti-angiogenic effects of Hypericum essential oils using the chorioallantoic membrane assay. Pharm Biol 52(1): 44$50,2014$.

25 Sui X, Chen R, Wang Z, Huang Z, Kong N, Zhang M, Han W, Lou F, Yang J, Zhang Q, Wang X, He C and Pan H (2013) Autophagy and chemotherapy resistance: a promising therapeutic target for cancer treatment. Cell Death Dis 4: e838, 2013.

26 Abou El Hassan MA, Verheul HM, Jorna AS, Schalkwijk C, van Bezu J, van der Vijgh WJ and Bast A: The new cardioprotector monohydroxyethylrutoside protects against doxorubicin-induced inflammatory effects in vitro. Br J Cancer 89(2): 357-362, 2003.

27 Woodley-Cook J, Shin LY, Swystun L, Caruso S, Beaudin S and Liaw PC: Effects of the chemotherapeutic agent doxorubicin on the protein $\mathrm{C}$ anticoagulant pathway. Mol Cancer Ther 5(12): 3303-3311, 2006.

28 Schnaper HW, Kleinman HK and Grant DS: Role of laminin in endothelial cell recognition and differentiation. Kidney Int 43(1): 20-25, 1993. 
29 Akeson AL, Brooks SK, Thompson FY and Greenberg JM: In vitro model for developmental progression from vasculogenesis to angiogenesis with a murine endothelial precursor cell line, MFLM-4. Microvasc Res 61(1): 75-86, 2001.

30 Goodwin AM: In vitro assays of angiogenesis for assessment of angiogenic and anti-angiogenic agents. Microvasc Res 74(2-3): 172-183, 2007.

$31 \mathrm{Li}$ Q, Yuan DM, Ma LH, Ma CH, Liu YF, Lv TF and Song Y: Chloroquine inhibits tumor growth and angiogenesis in malignant pleural effusion. Tumour Biol 37: 16249-16258, 2016.

32 Nishida N, Yano H, Nishida T, Kamura T, Kojiro M: Angiogenesis in cancer. Vasc Health Risk Manag 2(3): 213-219, 2006.

33 Kiriakidis S, Högemeier O, Starcke S, Dombrowski F, Hahne JC, Pepper M, Hem CJ and Wernert N: Novel tempeh (fermented soyabean) isoflavones inhibit in vivo angiogenesis in the chicken chorioallantoic membrane assay. Br J Nutr 93: 317323, 2005.

34 El-Kenawi AE, El-Remessy AB: Angiogenesis inhibitors in cancer therapy: mechanistic perspective on classification and treatment rationales. Br J Pharmacol 170(4): 712-729, 2013.

35 De Palma M, Biziato D and Petrova VT: Microenvironmental regulation of tumour angiogenesis. Nat Rev Cancer 17(8): 457474, 2017.
36 Potente M, Gerhardt $\mathrm{H}$ and Carmeliet P: Basic and therapeutic aspects of angiogenesis. Cell 146: 873-887, 2011.

$37 \mathrm{Yu}$ J, Wang C, Kong Q, Wu X, Lu JJ and Chen X: Recent progress in doxorubicin-induced cardiotoxicity and protective potential of natural products. Phytomedicine 40: 125-139, 2018.

38 Maragoudakis ME, Peristeris P, Missirlis E, Aletras A, Andriopoulou $P$ and Haralabopoulos G: Inhibition of angiogenesis by anthracyclines and titanocene dichloride. Ann NY Acad Sci 732(1): 280-293, 1994.

39 Liu F, Shang Y and Chen SZ: Chloroquine potentiates the anticancer effect of lidamycin on non-small cell lung cancer cells in vitro. Acta Pharmacol Sin 35(5): 645-652, 2014.

40 Maes H, Kuchnio A, Carmeliet P and Agostinis P: Chloroquine anticancer activity is mediated by autophagy-independent effects on the tumor vasculature. Mol Cell Oncol 3(1): e97009, 2016.

41 Bikfalvi A, Moenner M, Javerzat S, North S and Hagedorn M: Inhibition of angiogenesis and the angiogenesis/invasion shift. Biochem Soc Trans 39(6): 1560-1564, 2011.

Received April 2, 2018

Revised May 17, 2018

Accepted May 21, 2018 